\title{
Mean-Variance-CvaR Model of Multiportfolio Optimization via Linear Weighted Sum Method
}

\author{
Younes Elahi and Mohd Ismail Abd Aziz \\ Faculty of Science, Department of Mathematics, Universiti Teknologi Malaysia, 81310 Johor Bahru, Johor, Malaysia \\ Correspondence should be addressed to Younes Elahi; elahi.math@gmail.com
}

Received 16 July 2013; Accepted 22 January 2014; Published 30 March 2014

Academic Editor: Hao Shen

Copyright ( 2014 Y. Elahi and M. I. Abd Aziz. This is an open access article distributed under the Creative Commons Attribution License, which permits unrestricted use, distribution, and reproduction in any medium, provided the original work is properly cited.

\begin{abstract}
We propose a new approach to optimizing portfolios to mean-variance-CVaR (MVC) model. Although of several researches have studied the optimal MVC model of portfolio, the linear weighted sum method (LWSM) was not implemented in the area. The aim of this paper is to investigate the optimal portfolio model based on MVC via LWSM. With this method, the solution of the MVC model of portfolio as the multiobjective problem is presented. In data analysis section, this approach in investing on two assets is investigated. An MVC model of the multiportfolio was implemented in MATLAB and tested on the presented problem. It is shown that, by using three objective functions, it helps the investors to manage their portfolio better and thereby minimize the risk and maximize the return of the portfolio. The main goal of this study is to modify the current models and simplify it by using LWSM to obtain better results.
\end{abstract}

\section{Introduction}

In financial activity, a portfolio is a set of assets that allows somebody to choose from several choices in investing. The main problem in this area is to find out the optimal combination to distribute a given fund on a set of existing assets. Maximization of expected return and minimization of risk are two main aims of this problem [1].

Our goal is to develop the MVC model for portfolio with the weighted coefficients in which this approach is adaptive of the weighted sum method (WSM). One of the aims is to offer a practical method for the construction and optimize the proposed model, in order to produce the Pareto optimal solution. There are several researches that have been done on the optimal mean-variance-CVaR (MVC) model of portfolio but no much studies have been carried out in the area by using the linear weighted sum method (LWSM). This approach will give better insight into the multiportfolio process and the position of investing via finding the feasible solution set.

The paper is organized into five sections. In the next section, this paper presents the properties of multiobjective portfolio optimization based on mathematical approach and MVC model of portfolio optimization. Then, MVC model of portfolio based on LWSM will be presented. After that, the empirical example and some challenges will be shown to clarify the discussion. For this aim, an MVC model of the multiportfolio was implemented in MATLAB and tested on the presented problem. Finally, results and some future works are presented in the conclusion.

\section{MVC Model of Portfolio}

We face making many decisions in the real world. There are numerous methods to optimize them. The aim of this paper is to find the Pareto optimal solutions. However, in the theoretical problem cases, if Pareto set normally cannot be solved by an algorithm, then we try to approximate the Pareto sets [2].

For example, the multiobjective model for five objective functions $f_{1}, \ldots, f_{5}$ and some constraints can be shown by following model [3]:

$$
\operatorname{Max}\left[f_{1}(x), \ldots, f_{5}(x)\right]^{T}
$$




$$
\text { s.t. } \quad \sum_{i=1}^{M} x_{i}=1 \quad x_{i} \geq 0
$$

For $i=1, \ldots, M$.

MVC uses the three parameters (the expected value $(E)$, the variance $\left(\sigma^{2}\right)$, and the CVaR (conditional value at risk) at a specified confidence level $\alpha \in(0,1))$ for better modeling. The aim of the proposed model is to give a developed method for the solution [4].

We can obtain a better model of portfolio by replacing the three indexes instead of two parameters as usual so that it increases the efficiency of the model [5]. Several studies on portfolio optimizations via mathematical approaches have been investigated in the literature. To start the construction of this model, we consider some variables, terms, and objective functions as the following nomenclature [6].

Let

$N$ be the number of assets that are available;

$R_{x}=$ return (as a random variable) depending on a decision vector $x$ that belongs to a feasible set $A$;

$x_{i}=$ amount of investing in ith asset;

$\Omega=$ the feasible set of solutions or search space;

$s=$ a solution of problem;

$\mu_{i}$ the expected mean of the $i$ th asset;

$\sigma^{2}\left(R_{x}\right)=$ the variance belong to $R_{x}$;

$K$ the number of assets to invest $(K \leq N)$.

We define the variable

$z_{i}= \begin{cases}1 & \text { if the } i \text { th }(i=1, \ldots, N) \text { asset is chosen } \\ 0 & \text { otherwise }\end{cases}$

$x_{i}=$ money ratio $\left(0 \leq x_{i} \leq 1\right)$ invested in the $i$ th $(i=$ $1, \ldots, N)$ asset.

We consider the MVC model based on Aboulaich et al. method with following formula [4]:

$$
(P)\left\{\begin{array}{l}
\min \{\mathrm{CVaR},-E, \operatorname{var}\}, \\
\text { s.t } x \in\left\{\left(x_{1}, \ldots, x_{n}\right) \mid \sum_{j=1}^{n} x_{j}=1,\right. \\
\left.x_{j} \geq 0, \forall j \in\{1, \ldots, n\}\right\} .
\end{array}\right.
$$

We note that, for random variable, the value of variance is calculated by the following formula:

$$
\sigma^{2}\left(R_{x}\right)=E\left[\left(R_{x}-E\left(R_{x}\right)\right)^{2}\right] .
$$

To calculate the variance, let $R_{x}=R_{1} x_{1}+\cdots+R_{n} x_{n}$, so we have

$$
\sigma^{2}\left(R_{x}\right)=\sum_{i=1}^{n} \sum_{j=1}^{n} x_{i} x_{j} \delta_{i j}
$$

where $\delta_{i j}=\operatorname{cov}\left(R_{i}, R_{j}\right)$. In addition, in the model above, preferring the random variable $R_{x}$ than random variable $R_{y}$ is as the following conditions:

$$
\begin{gathered}
E\left(R_{x}\right) \geq E\left(R_{y}\right) ; \quad \sigma^{2}\left(R_{x}\right) \leq \sigma^{2}\left(R_{y}\right), \\
\operatorname{CVaR}\left(R_{x}\right) \leq \operatorname{CVaR}\left(R_{y}\right),
\end{gathered}
$$

in which CVaR is calculated according to following theorem.

Proposition 1 (CVaR calculation and optimization). Let $R_{x}$ be a random variable depending on a decision vector $x$ that belongs to a feasible set $A$ and $\alpha \in(0,1)$. Consider the function

$$
F_{\alpha}(x, v)=\frac{1}{\alpha} E\left\{\left[-R_{x},+v\right]^{+}\right\}-v,
$$

where $[u]^{+}=u$ for $u \geq 0$ and $[u]^{+}=0$ for $u<0$. Then, according to a function of $v, F_{\alpha}$ is finite and continuous (hence convex) and

$$
\operatorname{CVaR}\left(F_{x}\right)=\min _{v \in R} F_{\alpha}(x, v) .
$$

In addition, the set consisting of the values of $v$ for which the minimum is attained, denoted by $A_{\alpha}(x)$, is a nonempty, closed, and bounded interval. Minimizing $C V a R_{\alpha}$ with respect to $x \in$ $A$ is equivalent to minimizing $F_{\alpha}$ with respect to $(x, v) \in A x R$ :

$$
\min _{x \in A} C V a R_{\alpha}\left(R_{x}\right)=\min _{(x, v) \in A x R} F_{\alpha}(x, v) .
$$

In addition, a pair $\left(x^{*}, v^{*}\right)$ minimizes the right-hand side if and only if $x^{*}$ minimize the left-hand side and $v^{*} \epsilon$ $A_{\alpha}\left(x^{*}\right) . C V a R_{\alpha}\left(R_{x}\right)$ is convex with respect to $x$ and $F_{\alpha}(x, v)$ is convex with respect to $(x, v)$.

Thus, if the set $A$ of feasible decision vectors is convex (which is the case for the basic version of the portfolio selection problem), and even if we impose a further lower limit on the expected return, minimizing $C V a R$ is a convex optimization problem [7].

On the other expression, MVC model denotes as the following:

$$
\begin{array}{ll}
\min \sigma^{2}(x) & \\
\text { subject to: } & F_{\alpha}(x, v) \leq z \\
& E(x) \geq d \\
& x \in A, \quad v \in R,
\end{array}
$$

where variables $=\left(x_{1}, \ldots, x_{n}\right) \in A \subset R^{n}, v \in R$, and set of feasible decision vectors denotes via $A$ that is a convex set. In the model above, there is one multiobjective system for portfolio optimization that consists of three objective functions. The first objective function is to minimize the conditional value at risk for portfolio optimization. The second objective function denotes minimizing the expected value of return of the portfolio. And the last objective function considers minimizing the variance of returns. This procedure shows that we can manage our capital to invest in several assets [5]. 
TABLE 1: Situation of two assets.

\begin{tabular}{lccc}
\hline & & Criteria & \\
Alt. & $C_{1}$ & $C_{2}$ & $C_{3}$ \\
& 0.10 & 0.05 & 0.30 \\
\hline $\mathbf{A}_{1}$ & 15 & 10 & 25 \\
$\mathbf{A}_{2}$ & 30 & 20 & 10 \\
\hline
\end{tabular}

\section{MVC Model of Portfolio Based on LWSM}

This method was introduced by Zadeh in 1963 and it is one of the main ways of solving the MO (see [8]). Three types of this method are used for multiobjective portfolio optimization (MPO) in previous research. They are weighted sum method, weight quadratic method, and weight quadratic variation.

One of the main ways of weighting methods is the weighted sum method. The aim of weighting method is the optimization of the objective functions that they arranged by linear combination (weighted sum). Different efficient solutions can be found by changing the weights of the objective functions [9].

The weighted sum method changes the MO problem with a single model of mathematical optimization problem. In the model of this method sum weighting coefficient $w_{i}$ multiply each objective function $f_{i}$ to make the structure of the objective function as follows: (note that normalization of those coefficients is not necessary)

$$
\begin{aligned}
& \min \sum_{i=1}^{k} \omega_{i} f_{i}(x) \\
& \text { s.t. } \quad x \in \Omega, \\
& \quad \text { where } \quad \omega_{i} \geq 0, \quad \forall i=1, \ldots, \quad k \sum_{i=1}^{k} \omega_{i}=1 .
\end{aligned}
$$

With convexity supposition, if $\omega_{i}>0, \forall i=1, \ldots, k$, then the solution of the above system is Pareto optimal. This means that if that system is convex then any Pareto optimal solution can be found [10]. There are three criteria to measure the weight. They are subjective preference of the decision makers, the variance measure, and the independence of criteria. Usually two methods can be used to achieve this aim: the equal weights and the rank-order weights $[11,12]$.

Example 2 (see [13]). Consider that a multiobjective problem includes three criteria, which are denoted by the same unit, and two alternatives. According to Table 1 let $W_{1}=$ $0.10, W_{2}=0.05$, and $W_{3}=0.30$. The values of $\alpha_{i j}$ are assumed to be as follows:

$$
A=\left[\begin{array}{lll}
15 & 10 & 25 \\
30 & 20 & 10
\end{array}\right]
$$

Therefore, the decision matrix for this MCDM problem is as follows.

Criteria: 0.10, 0.05, 0.30.

Alternatives: $\mathbf{A}_{1}, \mathbf{A}_{2}$.
With (10) we obtain data as follows:

$$
\begin{aligned}
\mathbf{A}_{1}(\text { WSM Score })= & 15 \times 0.10+10 \\
& \times 0.05+25 \times 0.30=2.75 .
\end{aligned}
$$

In the same ways,

$$
\begin{aligned}
\mathbf{A}_{2}(\text { WSM Score })= & 30 \times 0.10+20 \\
& \times 0.05+10 \times 0.30=7 .
\end{aligned}
$$

So the optimal case in the minimization situation is alternative $\mathbf{A}_{\mathbf{1}}$ because it has the lowest WSM score. We can write the alternative ranking as follows: $\mathbf{A}_{\mathbf{1}}<\mathbf{A}_{\mathbf{1}}$ (where "<" denotes "better than").

Now, we formulate the portfolio revision problem with transaction costs as a standard mathematical optimization problem with the mean-risk framework. We will employ the following notation.

$$
\begin{aligned}
& N \text { : number of risky assets } \\
& e \text { : vector with all entries equal to ones. }
\end{aligned}
$$

Consider the optimization problem with the normalized single objective as follows:

$$
\begin{array}{ll}
\text { Maximize: } & f(x)=\sum_{k=1}^{l} \omega_{k} Q_{k}^{0}(x), \\
\text { Subject to: } & x \in X=\left\{x \mid f_{i}(x) \geq 0, g_{i(x)}=0, \overline{1, l}\right\},
\end{array}
$$

where the weights are denoted by $\omega_{i}$, and

$$
\omega_{i} \geq 0, \quad \sum_{i=1}^{l} \omega_{i}=1, \quad i=\overline{1, l} .
$$

And the objective function of $k$ th objective function $Q_{k}(x)$ after normalization is denoted by $Q_{k}^{0}(x), k=\overline{1, l}$. Also we call $f_{i}(x), g_{i(x)}$ inequality and equality constraints, respectively. With LWSM approach, we have

$$
Q_{k}(x)=\sum_{i=1}^{l} a_{k i} x_{i}, \quad a_{k i} \in R .
$$

Therefore, normalized objective functions have the following form:

$$
Q_{k}^{0}(x)=\frac{Q_{k}(x)}{S_{k}}=\frac{a_{k 1}}{S_{k}} x_{1}+\cdots+\frac{a_{k n}}{S_{k}} x_{n},
$$

in which case the floating-point values $S_{k}$ are evaluated in the following way:

$$
S_{k}=\sum_{j=1}^{n}\left|a_{k j}\right| \neq 0 \text {. }
$$

Obviously, in many practical problems, the objective functions are represented by various measure units (e.g., if 
$Q_{1}$ is measured in kilos, $Q_{2}$ in seconds, etc.). For this reason the objective function normalization is required. It is now obvious that the coefficients have values of the segment $[0,1]$. Denote that we now have the linear programming problem

$$
\text { Maximize: } \begin{aligned}
f(x)= & \sum_{k=1}^{l} \omega_{k} \frac{Q_{k}(x)}{S_{k}}=\omega_{1} \frac{a_{k 1}}{S_{k}} x_{1} \\
& +\cdots+\omega_{n} \frac{a_{k n}}{S_{k}} x_{n},
\end{aligned}
$$

Subject to: $x \in X$.

The following theorem gives the practical criteria for the detection of some Pareto optimal solutions of problem (2.4). Yu et al. [14] presented mean-CVaR model of portfolio optimization based on LWSM in which they assumed that the return of the portfolio is based on multi-t-distribution and they obtained the model for

$$
\max (u(w))=\max \left(\alpha_{1} E(R)-\alpha_{2} \mathrm{CVaR}\right)
$$

as follows:

$$
\begin{aligned}
& \max 0.5\left(w^{T} \mu\right)+0.5\left(\alpha+\frac{1}{m(1-0.5)} \sum_{k=1}^{m} u_{k}\right), \\
& \text { s.t } \begin{cases}w^{T} \mu+\alpha+u_{k} \geq 0 & u_{k} \geq 0 \\
\sum_{i=1}^{n} w_{i}=1 & 0 \leq w_{i} \leq 1 .\end{cases}
\end{aligned}
$$

There are some conditions for coefficients that are used in WSM. For example, the sum of them equal 1 and they are strictly positive. In addition to LWSM they must be normalized. With this idea, the multiportfolio optimization problem is as follows:

$$
\begin{aligned}
& \min (u(w))=\min \left(\alpha_{1} \sigma^{2}(R)-\alpha_{2} E(R)+\alpha_{3} \mathrm{CVaR}\right) \\
& \text { Subject to: } x \in A,
\end{aligned}
$$

where $\alpha_{1}, \alpha_{2}$, and $\alpha_{3}$ are strictly positive and $A$ is as the above assumptions. Since all objective functions on MVC model are convex, we can define the model above to find the solution as the Pareto optimal set [15]. For instance, if we consider $\alpha_{1}=1, \alpha_{2}=0$, and $\alpha_{3}=0$ the problem turns to minimize the variance of the portfolio [5].

Now, according to (P2) as the multiobjective optimization problem, LWSM will be used to solve it as in the following procedure.

Step 1. Construct the problem based on

$$
\min (u(w))=\min \left(\alpha_{1} \sigma^{2}(R)-\alpha_{2} E(R)+\alpha_{3} \mathrm{CVaR}\right) .
$$

There are several positions of investing for investor who can manage his/her budget which is related to the amount of $\alpha_{1}, \alpha_{2}$, and $\alpha_{3}$. This note creates several cases for investors to increase the return or decrease the risk of a portfolio. The risk (or return) has an inverse (direct) relation with those coefficients. The LWSM leads to following procedure for MVC model of portfolio.
Step 2. Consider

$$
\begin{gathered}
\min \alpha_{1}\left(w^{T} \sigma w\right)-\alpha_{2}\left(w^{T} \mu\right) \\
+\alpha_{3}\left(\alpha+\frac{1}{m(1-\beta)} \sum_{k=1}^{m} u_{k}\right) \\
\text { s.t } \begin{cases}w^{T} \mu+\alpha+u_{k} \geq 0 & u_{k} \geq 0 \\
\sum_{i=1}^{n} w_{i}=1 & 0 \leq w_{i} \leq 1,\end{cases}
\end{gathered}
$$

where $\alpha, \beta$ are the belief degree and specific parameter, respectively. In this paper, it is considered that $\beta=0.5$. In addition, according to $\sum_{i=1}^{n} \alpha_{i}=1$ we have $1-\left(\alpha_{1}+\alpha_{2}\right)=\alpha_{3}$; we rewrite problem of (18) as follows:

$$
\begin{array}{r}
\min \alpha_{1}\left(w^{T} \sigma w\right)-\alpha_{2}\left(w^{T} \mu\right)+\left(1-\left(\alpha_{1}+\alpha_{2}\right)\right) \\
\quad \times\left(\alpha+\frac{1}{m(1-0.5)} \sum_{k=1}^{m} u_{k}\right), \\
\text { s.t } \begin{cases}w^{T} \mu+\alpha+u_{k} \geq 0 & u_{k} \geq 0 \\
\sum_{i=1}^{n} w_{i} & 0 \leq w_{i} \leq 1,\end{cases}
\end{array}
$$

where the belief degree of $\alpha$ set is equal to 0.95 . In addition, in CVaR formula after linearization $[16,17]$ we have

$$
\widetilde{F}_{\beta}(w, \alpha)=\alpha+\frac{1}{m(1-\beta)} \sum_{k=1}^{m}\left[f\left(w, y_{k}\right)+\alpha\right]^{+},
$$

and $f\left(w, y_{k}\right)$ is the loss function which can be defined [16] by $f\left(w, y_{k}\right)=-w^{T} y_{k}$. We can rewrite (19) according to the assumptions above as follows:

$$
\begin{aligned}
& \min \alpha_{1}\left(w^{T} \sigma w\right)-\alpha_{2}\left(w^{T} \mu\right)+\left(1-\left(\alpha_{1}+\alpha_{2}\right)\right) \\
& \quad \times\left(0.95+\frac{1}{m(1-0.5)} \sum_{k=1}^{m}\left[-w^{T} y_{k}+0.95\right]^{+}\right), \\
& \text {s.t }\left\{\begin{array}{l}
{[u]^{+}=\max \{u, 0\}} \\
\sum_{i=1}^{n} w_{i}=1 \quad 0 \leq w_{i} \leq 1,
\end{array}\right.
\end{aligned}
$$

where $\mu_{k}$ is return of $k$ th asset. In the model above, we consider $m=2$ and we have

$$
\begin{aligned}
& \min \alpha_{1}\left(w^{T} \sigma w\right)-\alpha_{2}\left(w^{T} \mu\right)+\left(1-\left(\alpha_{1}+\alpha_{2}\right)\right) \\
& \quad \times\left(0.95+\frac{1}{2(1-0.5)} 2\left(\left[-w^{T} y_{k}+0.95\right]^{+}\right)\right), \\
& \text {s.t }\left\{\begin{array}{l}
{[u]^{+}=\max \{u, 0\}} \\
\sum_{i=1}^{n} w_{i}=1 \quad 0 \leq w_{i} \leq 1 .
\end{array}\right.
\end{aligned}
$$




\section{Empirical Research and Challenges}

In order to evaluate the model above to find the portfolio optimization based on LWSM we consider two assets whose mean return $\mu$ and covariance matrix $\sigma$ are as the following data (data from $[14,16])$ :

$$
\begin{gathered}
\mu=\left(\begin{array}{c}
0.06760987 \\
0.034191
\end{array}\right), \\
\sigma=\left(\begin{array}{cc}
0.058884101 & 0.000464043 \\
0.000464043 & 0.0003643663
\end{array}\right) .
\end{gathered}
$$

According to the data above and the model of (29) we have

$$
\min Z=\min \alpha_{1}\left(z_{1}\right)-\alpha_{2}\left(z_{2}\right)+\left(1-\left(\alpha_{1}+\alpha_{2}\right)\right)\left(z_{3}\right),
$$

where

$$
\begin{array}{rl}
z_{1}= & \delta_{p}^{2}=w^{T} \sigma w= \\
= & \left(0.058884101 * w_{1}+0.000464043 * w_{2}\right) * w_{1} \\
& +\left(0.000464043 * w_{1}+0.0003643663 * w_{2}\right) * w_{2}, \\
& z_{2}=\mu_{p}=w^{T} \mu=0.06760987 w_{1}+0.034191 w_{2}, \\
z_{3}= & \left(0.95+\frac{1}{2(1-0.5)} 2\left(\left[-w^{T} \mu-0.95\right]^{+}\right)\right) \\
= & 0.95+\frac{1}{2(0.5)} \\
& \times 2\left(\left[-\left(0.06760987 w_{1}+0.034191 w_{2}\right)+0.95\right]^{+}\right), \\
{[u]^{+}=\max \{u, 0\} \sum_{i=1}^{n} w_{i}} & 0 \leq w_{i} \leq 1 .
\end{array}
$$

Now, we consider this proposed model for $\alpha_{1}=0.25, \alpha_{2}=$ 0.25 . Therefore, we have

$$
\begin{gathered}
\min Z=\min \left(0.01472102525 * w_{1}^{2}\right. \\
+0.00058005375 * w_{1} * w_{2} \\
\left.+0.0003643663 * w_{2}^{2}\right) \\
-\left(0.0169024675 w_{1}+0.00854775 w_{2}\right) \\
+\left(0.475+\left(\left[-\left(0.06760987 w_{1}+0.034191 w_{2}\right)\right.\right.\right. \\
\text { s.t }\left\{\begin{array}{c}
{[u]^{+}=\max \{u, 0\}} \\
\sum_{i=1}^{n} w_{i}=1,
\end{array} \quad 0 \leq w_{i} \leq 1 .\right.
\end{gathered}
$$

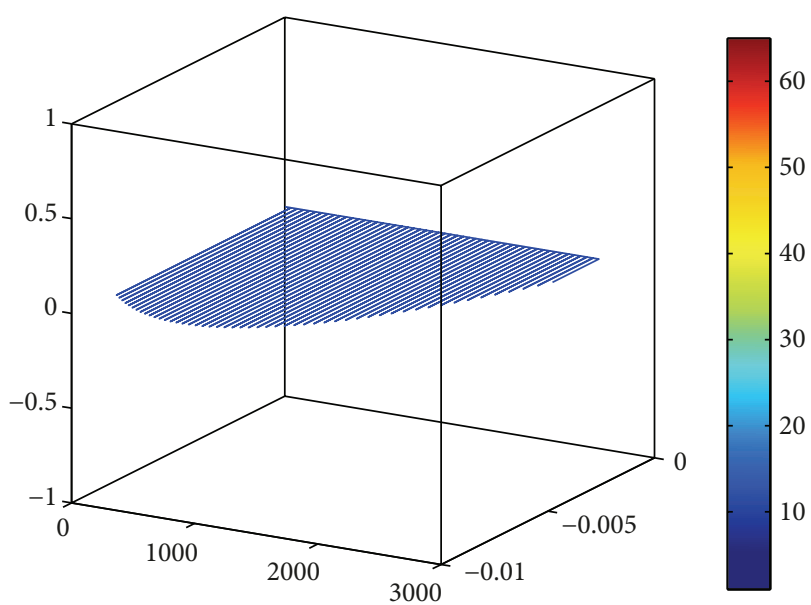

FIGURE 1: Mean-variance model of portfolio via LWSM.

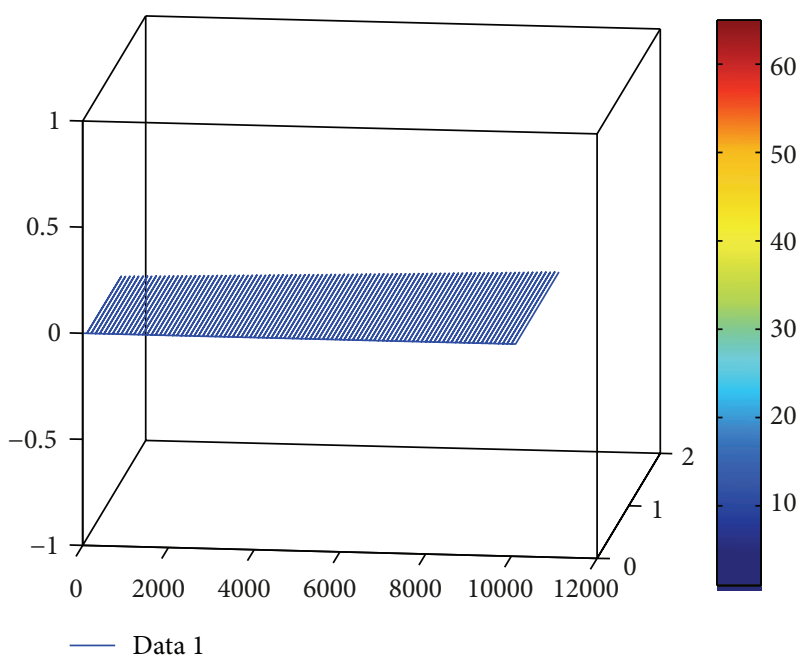

FIGURE 2: MVC model of portfolio via LWSM.

In order to compare the performance of mean-variance model and MVC model of multiportfolio optimization, the minimum functions are demonstrated via MATLABsoftware, as Figures 1 and 2 show the results of LWSM method for two models, respectively.

Comparative performance was measured using a MATLAB software, and performance was shown in Figures 1 and 2 which are called the mean-variance and MVC model of multiportfolio, respectively.

In summary, we compare the performance of MVC model of multiportfolio with LWSM technique and other models like mean-variance approaches. The mean-variance minimization function and the MVC model are as described in Figures 1 and 2, respectively.

Table 2 is arranged to illustrate the results of MVC model of portfolio and refer to two assets by Matlab by this approach.

As shown in the above results, we tested two assets via proposed model and we presented the results of that as Table 2 which included 20 results from our proposed model by MATLAB software. It showed, except for two obvious cases 
TABLE 2: Results of MVC model of portfolio refer to two assets (see Section 4) by MATLAB.

\begin{tabular}{|c|c|c|}
\hline$w_{1}=0.000000$ & $w_{2}=1.000000$ & $z=1.083819$ \\
\hline$w_{1}=0.010000$ & $w_{2}=0.990000$ & $z=1.086554$ \\
\hline$w_{1}=0.020000$ & $w_{2}=0.980000$ & $z=1.089293$ \\
\hline$w_{1}=0.030000$ & $w_{2}=0.970000$ & $z=1.092035$ \\
\hline$w_{1}=0.040000$ & $w_{2}=0.960000$ & $z=1.094779$ \\
\hline$w_{1}=0.050000$ & $w_{2}=0.950000$ & $z=1.097527$ \\
\hline$w_{1}=0.060000$ & $w_{2}=0.940000$ & $z=1.100278$ \\
\hline$w_{1}=0.070000$ & $w_{2}=0.930000$ & $z=1.103031$ \\
\hline$w_{1}=0.080000$ & $w_{2}=0.920000$ & $z=1.105788$ \\
\hline$w_{1}=0.090000$ & $w_{2}=0.910000$ & $z=1.108547$ \\
\hline$w_{1}=0.100000$ & $w_{2}=0.900000$ & $z=1.111310$ \\
\hline$w_{1}=0.110000$ & $w_{2}=0.890000$ & $z=1.114075$ \\
\hline$w_{1}=0.120000$ & $w_{2}=0.880000$ & $z=1.116844$ \\
\hline$w_{1}=0.130000$ & $w_{2}=0.870000$ & $z=1.119615$ \\
\hline$w_{1}=0.140000$ & $w_{2}=0.860000$ & $z=1.122389$ \\
\hline$w_{1}=0.150000$ & $w_{2}=0.850000$ & $z=1.125167$ \\
\hline$w_{1}=0.160000$ & $w_{2}=0.840000$ & $z=1.127947$ \\
\hline$w_{1}=0.170000$ & $w_{2}=0.830000$ & $z=1.130730$ \\
\hline$w_{1}=0.180000$ & $w_{2}=0.820000$ & $z=1.133517$ \\
\hline$w_{1}=0.190000$ & $w_{2}=0.810000$ & $z=1.136306$ \\
\hline$w_{1}=0.200000$ & $w_{2}=0.800000$ & $z=1.139098$ \\
\hline$w_{1}=0.210000$ & $w_{2}=0.790000$ & $z=1.141893$ \\
\hline$w_{1}=0.220000$ & $w_{2}=0.780000$ & $z=1.144691$ \\
\hline$w_{1}=0.230000$ & $w_{2}=0.770000$ & $z=1.147493$ \\
\hline$w_{1}=0.240000$ & $w_{2}=0.760000$ & $z=1.150297$ \\
\hline$w_{1}=0.250000$ & $w_{2}=0.750000$ & $z=1.153104$ \\
\hline$w_{1}=0.260000$ & $w_{2}=0.740000$ & $z=1.155914$ \\
\hline$w_{1}=0.270000$ & $w_{2}=0.730000$ & $z=1.158727$ \\
\hline$w_{1}=0.280000$ & $w_{2}=0.720000$ & $z=1.161543$ \\
\hline$w_{1}=0.290000$ & $w_{2}=0.710000$ & $z=1.164362$ \\
\hline$w_{1}=0.300000$ & $w_{2}=0.700000$ & $z=1.167184$ \\
\hline$w_{1}=0.310000$ & $w_{2}=0.690000$ & $z=1.170009$ \\
\hline$w_{1}=0.320000$ & $w_{2}=0.680000$ & $z=1.172837$ \\
\hline$w_{1}=0.330000$ & $w_{2}=0.670000$ & $z=1.175668$ \\
\hline$w_{1}=0.340000$ & $w_{2}=0.660000$ & $z=1.178501$ \\
\hline$w_{1}=0.350000$ & $w_{2}=0.650000$ & $z=1.181338$ \\
\hline$w_{1}=0.360000$ & $w_{2}=0.640000$ & $z=1.184178$ \\
\hline$w_{1}=0.370000$ & $w_{2}=0.630000$ & $z=1.187021$ \\
\hline$w_{1}=0.380000$ & $w_{2}=0.620000$ & $z=1.189867$ \\
\hline$w_{1}=0.390000$ & $w_{2}=0.610000$ & $z=1.192715$ \\
\hline$w_{1}=0.400000$ & $w_{2}=0.600000$ & $z=1.195567$ \\
\hline$w_{1}=0.410000$ & $w_{2}=0.590000$ & $z=1.198422$ \\
\hline$w_{1}=0.420000$ & $w_{2}=0.580000$ & $z=1.201279$ \\
\hline$w_{1}=0.430000$ & $w_{2}=0.570000$ & $z=1.204140$ \\
\hline$w_{1}=0.440000$ & $w_{2}=0.560000$ & $z=1.207004$ \\
\hline$w_{1}=0.450000$ & $w_{2}=0.550000$ & $z=1.209870$ \\
\hline$w_{1}=0.460000$ & $w_{2}=0.540000$ & $z=1.212740$ \\
\hline$w_{1}=0.470000$ & $w_{2}=0.530000$ & $z=1.215612$ \\
\hline$w_{1}=0.480000$ & $w_{2}=0.520000$ & $z=1.218488$ \\
\hline$w_{1}=0.490000$ & $w_{2}=0.510000$ & $z=1.221366$ \\
\hline
\end{tabular}

TABLE 2: Continued.

\begin{tabular}{|c|c|c|}
\hline$w_{1}=0.500000$ & $w_{2}=0.500000$ & $z=1.224248$ \\
\hline$w_{1}=0.510000$ & $w_{2}=0.490000$ & $z=1.227132$ \\
\hline$w_{1}=0.520000$ & $w_{2}=0.480000$ & $z=1.230019$ \\
\hline$w_{1}=0.530000$ & $w_{2}=0.470000$ & $z=1.232910$ \\
\hline$w_{1}=0.540000$ & $w_{2}=0.460000$ & $z=1.235803$ \\
\hline$w_{1}=0.550000$ & $w_{2}=0.450000$ & $z=1.238699$ \\
\hline$w_{1}=0.560000$ & $w_{2}=0.440000$ & $z=1.241599$ \\
\hline$w_{1}=0.570000$ & $w_{2}=0.430000$ & $z=1.244501$ \\
\hline$w_{1}=0.580000$ & $w_{2}=0.420000$ & $z=1.247406$ \\
\hline$w_{1}=0.590000$ & $w_{2}=0.410000$ & $z=1.250314$ \\
\hline$w_{1}=0.600000$ & $w_{2}=0.400000$ & $z=1.253225$ \\
\hline$w_{1}=0.610000$ & $w_{2}=0.390000$ & $z=1.256140$ \\
\hline$w_{1}=0.620000$ & $w_{2}=0.380000$ & $z=1.259057$ \\
\hline$w_{1}=0.630000$ & $w_{2}=0.370000$ & $z=1.261977$ \\
\hline$w_{1}=0.640000$ & $w_{2}=0.360000$ & $z=1.264900$ \\
\hline$w_{1}=0.650000$ & $w_{2}=0.350000$ & $z=1.267826$ \\
\hline$w_{1}=0.660000$ & $w_{2}=0.340000$ & $z=1.270755$ \\
\hline$w_{1}=0.670000$ & $w_{2}=0.330000$ & $z=1.273687$ \\
\hline$w_{1}=0.680000$ & $w_{2}=0.320000$ & $z=1.276622$ \\
\hline$w_{1}=0.690000$ & $w_{2}=0.310000$ & $z=1.279560$ \\
\hline$w_{1}=0.700000$ & $w_{2}=0.300000$ & $z=1.282501$ \\
\hline$w_{1}=0.710000$ & $w_{2}=0.290000$ & $z=1.285445$ \\
\hline$w_{1}=0.720000$ & $w_{2}=0.280000$ & $z=1.288392$ \\
\hline$w_{1}=0.730000$ & $w_{2}=0.270000$ & $z=1.291341$ \\
\hline$w_{1}=0.740000$ & $w_{2}=0.260000$ & $z=1.294294$ \\
\hline$w_{1}=0.750000$ & $w_{2}=0.250000$ & $z=1.297250$ \\
\hline$w_{1}=0.760000$ & $w_{2}=0.240000$ & $z=1.300209$ \\
\hline$w_{1}=0.770000$ & $w_{2}=0.230000$ & $z=1.303170$ \\
\hline$w_{1}=0.780000$ & $w_{2}=0.220000$ & $z=1.306135$ \\
\hline$w_{1}=0.790000$ & $w_{2}=0.210000$ & $z=1.309103$ \\
\hline$w_{1}=0.800000$ & $w_{2}=0.200000$ & $z=1.312074$ \\
\hline$w_{1}=0.810000$ & $w_{2}=0.190000$ & $z=1.315047$ \\
\hline$w_{1}=0.820000$ & $w_{2}=0.180000$ & $z=1.318024$ \\
\hline$w_{1}=0.830000$ & $w_{2}=0.170000$ & $z=1.321003$ \\
\hline$w_{1}=0.840000$ & $w_{2}=0.160000$ & $z=1.323986$ \\
\hline$w_{1}=0.850000$ & $w_{2}=0.150000$ & $z=1.326971$ \\
\hline$w_{1}=0.860000$ & $w_{2}=0.140000$ & $z=1.329960$ \\
\hline$w_{1}=0.870000$ & $w_{2}=0.130000$ & $z=1.332951$ \\
\hline$w_{1}=0.880000$ & $w_{2}=0.120000$ & $z=1.335946$ \\
\hline$w_{1}=0.890000$ & $w_{2}=0.110000$ & $z=1.338943$ \\
\hline$w_{1}=0.900000$ & $w_{2}=0.100000$ & $z=1.341944$ \\
\hline$w_{1}=0.910000$ & $w_{2}=0.090000$ & $z=1.344947$ \\
\hline$w_{1}=0.920000$ & $w_{2}=0.080000$ & $z=1.347953$ \\
\hline$w_{1}=0.930000$ & $w_{2}=0.070000$ & $z=1.350963$ \\
\hline$w_{1}=0.940000$ & $w_{2}=0.060000$ & $z=1.353975$ \\
\hline$w_{1}=0.950000$ & $w_{2}=0.050000$ & $z=1.356990$ \\
\hline$w_{1}=0.960000$ & $w_{2}=0.040000$ & $z=1.360008$ \\
\hline$w_{1}=0.970000$ & $w_{2}=0.030000$ & $z=1.363030$ \\
\hline$w_{1}=0.980000$ & $w_{2}=0.020000$ & $z=1.366054$ \\
\hline$w_{1}=0.990000$ & $w_{2}=0.010000$ & $z=1.369081$ \\
\hline$w_{1}=1.000000$ & $w_{2}=0.000000$ & $z=1.372111$ \\
\hline
\end{tabular}


$\left(w_{1}=0\right.$ and $w_{2}=1 ; w_{1}=1$ and $\left.w_{2}=0\right)$, that the minimum situation of MVC model of multiportfolio will occurr on $w_{1}=$ 0.01 and $w_{2}=0.99$. The results illustrate that the proposed method is better for investing a small amount of assets such as the above two assets.

Despite of the several advantages, this method has some disadvantages which one of them is the weights $\alpha_{1}, \alpha_{2}$, and $\alpha_{3}$ that are hard to understand [5]. The other one is how to use the Simplex method to solve the weighted sum problem. The solution of this problem is using other techniques (e.g., interior-point methods) [10].

\section{Conclusion}

In this investigation, we consider three objective functions to model portfolio optimization with LWSM. In addition, we propose a linear weighted sum method to solve the MCV model of portfolio optimization. Also, with empirical example and MATLAB software, we evaluated proposed model. Our example includes two assets for investing. However, it is flexible to choose more assets and find the Pareto optimal according to procedures that are used in this paper.

The contribution of this study is a presentation of MVC model of portfolio optimization based on LWSM. The empirical examples have shown that using the three objective functions to make a powerful multi-portfolio model. We have described the MVC model of multiportfolio optimization that it is extended of the model presented by Yu et al. [14]. In addition, we showed that the MVC model generally covers both mean-variance and mean-CVaR models of portfolio optimization (with changing the coefficients of $\alpha_{i}, i=\overline{1,3}$ ).

Furthermore, this paper has given new insight into the multiobjective decision making process via WSM. A hybrid of this method and other techniques such as interior-point methods can be a good idea for future researchers.

To sum up, with using three objective functions, it helps the investors to manage their portfolio better and thereby minimize the risk and maximize the return of the portfolio. Also, using LWSM dues to modify the current models and simplify it to obtain better results. The same approach can also be used for other multiobjective optimization model and investing applications.

\section{Conflict of Interests}

The authors declare that there is no conflict of interests regarding the publication of this paper.

\section{Acknowledgment}

Research reported in this publication was supported by Universiti Teknologi Malaysia under grant number 4B112 (Research Management Centre, Universiti Teknologi Malaysia).

\section{References}

[1] Y. Elahi and A. A. Mohd-Ismail, "Multi-objectives portfolio optimization, challenges and opportunities for islamic approach," Australian Journal of Basic and Applaied Sicence, vol. 6, no. 10, pp. 297-302, 2012.

[2] I. Radziukynienè and A. Žilinskas, "Evolutionary methods for multi-objective portfolio optimization," in Proceedings of the World Congress on Engineering, 2008.

[3] C. Chiranjeevi and V. N. Sastry, "Multi objective portfolio optimization models and its solution using genetic algorithms," in Proceedings of the International Conference on Computational Intelligence and Multimedia Applications (ICCIMA '07), December 2007.

[4] R. Aboulaich, R. Ellaia, and S. El Moumen, "The meanvariance-CVaR model for portfolio optimization modeling using a multi-objective approach based on a hybrid method," Mathematical Modelling of Natural Phenomena, vol. 5, no. 7, pp. 103-108, 2010.

[5] D. Roman, K. Darby-Dowman, and G. Mitra, "Mean-risk models using two risk measures: a multi-objective approach," Quantitative Finance, vol. 7, no. 4, pp. 443-458, 2007.

[6] R. Armañanzas and J. A. Lozano, "A multiobjective approach to the portfolio optimization problem," in Proceedings of the IEEE Congress on Evolutionary Computation (CEC '05), September 2005.

[7] D. Roman, K. Darby-Dowman, and G. Mitra, "Portfolio construction based on stochastic dominance and target return distributions," Mathematical Programming, vol. 108, no. 2, pp. 541-569, 2006.

[8] L. Zadeh, "Optimality and non-scalar-valued performance criteria," IEEE Transactions on Automatic Control, vol. 8, no. 1, pp. 59-60, 1963.

[9] K. Kirytopoulos, V. Leopoulos, G. Mavrotas, and D. Voulgaridou, "Multiple sourcing strategies and order allocation: an ANP-AUGMECON meta-model," Supply Chain Management, vol. 15, no. 4, pp. 263-276, 2010.

[10] O. Grodzevich and O. Romanko, "Normalization and other topics in multi-objective optimization," 2006.

[11] J. Jia, G. W. Fischer, and J. S. Dyer, "Attribute weighting methods and decision quality in the presence of response error: a simulation study," Journal of Behavioral Decision Making, vol. 11, no. 2, pp. 85-105, 1998.

[12] B. Sawik, "A reference point approach to bi-objective dynamic portfolio optimization," Decision Making in Manufacturing and Services, vol. 3, no. 1-2, pp. 73-85, 2009.

[13] O. Romanko, A. Ghaffari-Hadigheh, and T. Terlaky, "Multiobjective optimization via parametric optimization: models, algorithms, and applications," Modeling and Optimization, pp. 77-119, 2012.

[14] X. Yu, Y. Tan, L. Liu, and W. Huang, "The optimal portfolio model based on mean-CvaR with linear weighted sum method," in Fifth International Joint Conference on Computational Sciences and Optimization (CSO '12), pp. 82-84, June 2012.

[15] J. Jahn, "Some characterizations of the optimal solutions of a vector optimization problem," Operations-Research-Spektrum, vol. 7, pp. 7-17, 1985.

[16] X. Yu, H. Sun, and G. Chen, "The optimal portfolio model based on mean-CVaR," Journal of Mathematical Finance, vol. 1, no. 3, pp. 132-134, 2011.

[17] S. Uryasev, "Conditional value-at-risk: optimization algorithms and applications," in Proceedings of the IEEE/IAFE/INFORNS 6th Conference on Computational Intelligence for Financial Engineering (CIFEr '00), pp. 49-57, March 2000. 


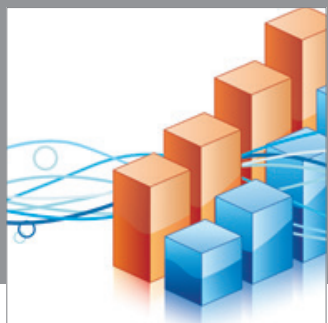

Advances in

Operations Research

mansans

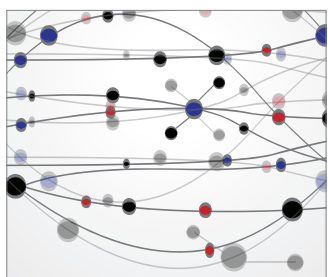

The Scientific World Journal
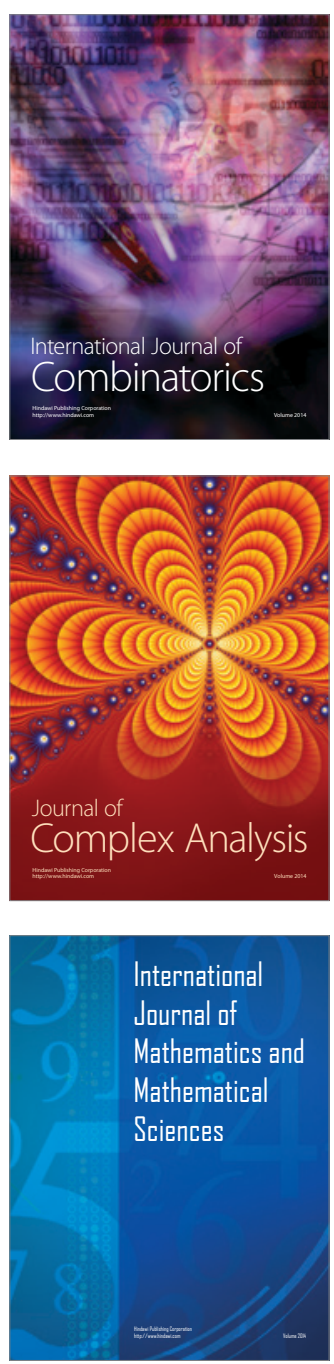
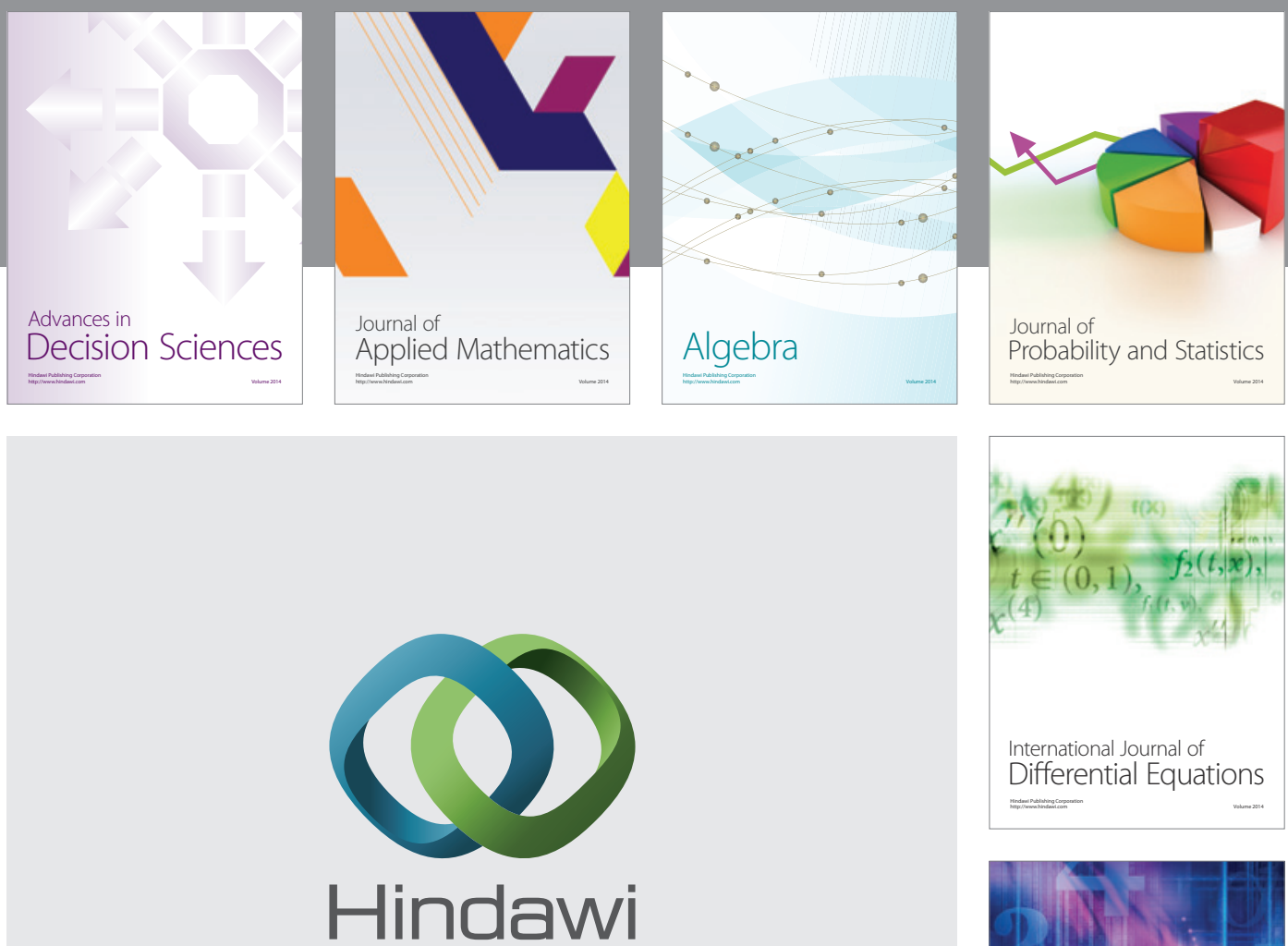

Submit your manuscripts at http://www.hindawi.com
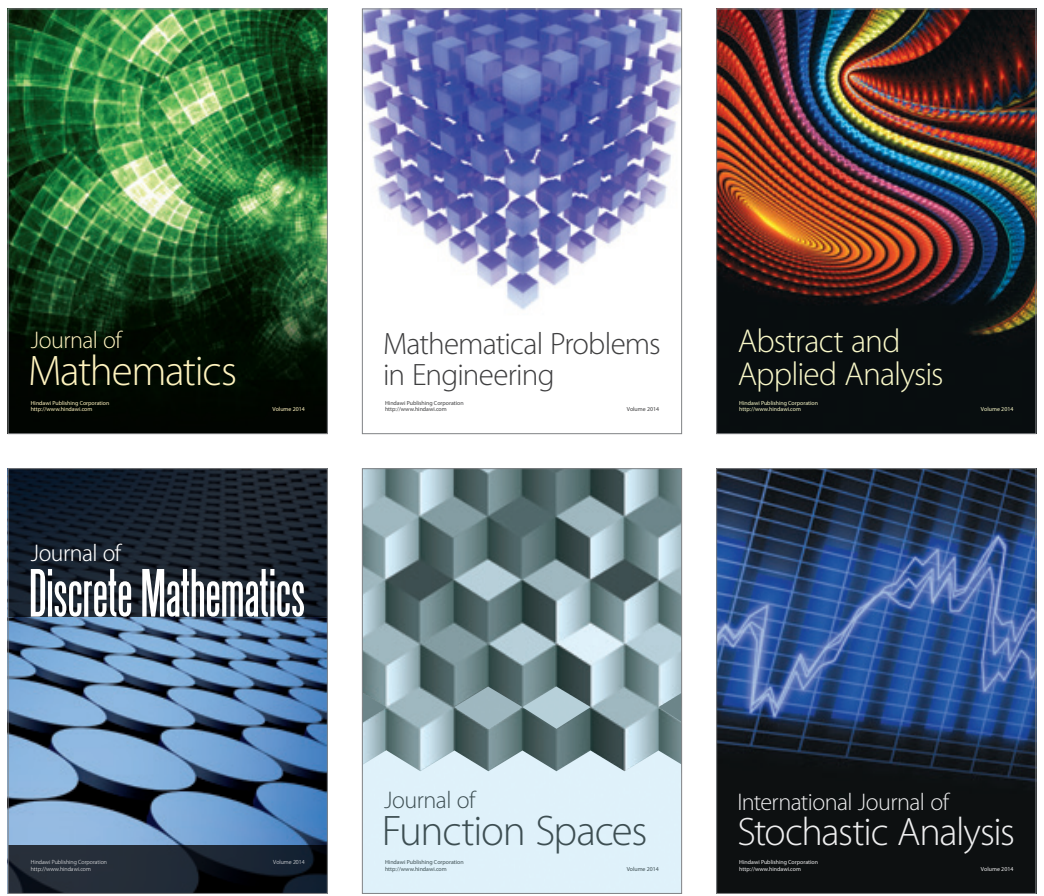

Journal of

Function Spaces

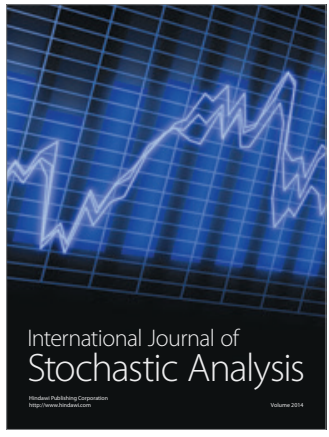

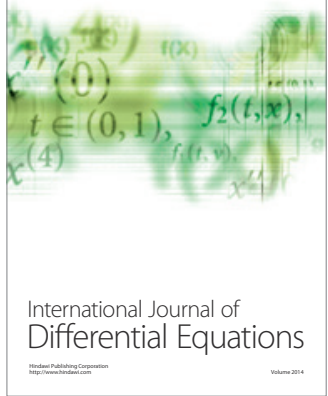
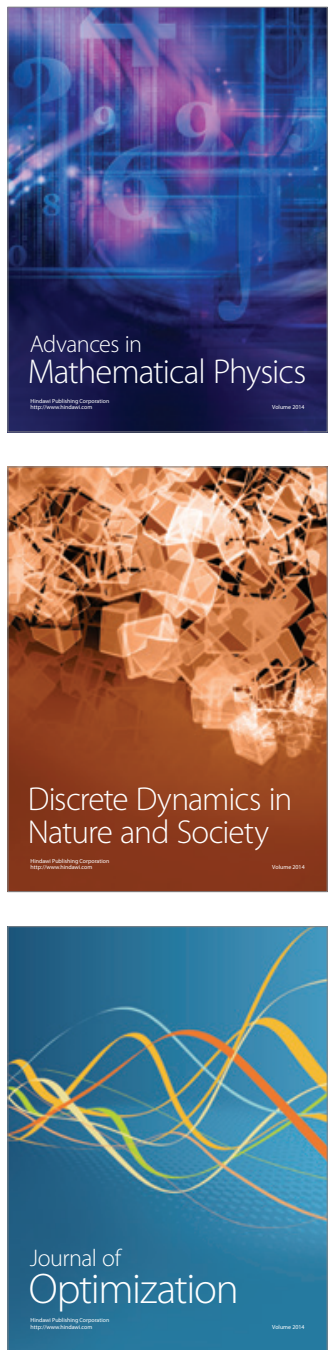